\title{
Three-dimensional chromospheric magnetic field configurations based on photospheric-vector and chromospheric-multi-level longitudinal-magnetic field observations
}

\author{
S. Cuperman, C. Bruma, and D. Heristchi \\ DASOP, Observatoire de Paris, Section Meudon, F-92195 Meudon Principal Cedex, France
}

Received March 14; accepted July 22, 1996

\begin{abstract}
The three-dimensional (3D) reconstruction of magnetic configurations above the photosphere is considered within the framework of the nonlinear force-free-field (FFF) model. The physical- computational algorithm proposed and tested incorporates, for the first time, the following basic features: 1) Both photospheric vector field, $\boldsymbol{B}(x, y, 0)$ and chromospheric line of sight field component, $B_{z}(x, y, z)$ data are utilized; this reduces significantly the degree of ill-posedness characterizing the Cauchy problem corresponding to the case when only $\boldsymbol{B}(x, y, 0)$ - values are used as boundary conditions. 2) A high-order, very efficient computational algorithm is developed and used: horizontal derivatives are evaluated by 14 - terms formulas in 14 different forms, selected such as to provide optimal computational accuracy; the vertical integration is achieved by the use of "moving" 10 - term formulas expressed in terms of 10 derivatives and the first $B_{i}(x, y, z)$ values $(i=x, y, z) .3)$ At neutral points, where inherent computational singularities in the values of the FFFfunction $\alpha$ arise, rather than using smoothing techniques based on four-neighbouring- values averages, suitable procedures ensuring continuity are developed and used. The overall result of the incorporation of these novel features is an improvement by orders of magnitude of the accuracy with which the chromospheric fields are reconstructed in the case in which one uses (i) only $\boldsymbol{B}(x, y, 0)$ - values as boundary conditions and (ii) relative simple computational formulas and smoothing techniques; at $\bar{z}=20$, $\Delta B_{i} / B_{i}<10^{-3}$ ! The elimination/minimization of measurement errors as well as the fitting of the corrected date to FFF-model-states is also discussed.
\end{abstract}

Key words: MHD - methods: numerical - Sun: magnetic fields

Send offprint requests to: S. Cuperman

\section{Introduction}

The solar chromospheric and coronal magnetic fields play a major role in the physics of the solar atmosphere. So far, these magnetic fields were determined by the extrapolation of observed photospheric magnetic fields upon assuming either (1) a potential, current-free model and using (only) the line of sight component or (ii) a force-free field (FFF) model $(\boldsymbol{J}=\alpha \boldsymbol{B}, \alpha=\alpha(r))$, and using all three components of the photospheric field. Various analytical or/and numerical methods for the determination of the magnetic field in half the space above the photosphere have been suggested or/and implemented. (See, e.g., the reviews by Sakurai 1989 and Gary 1990; also, the papers by Schmidt 1964; Semel 1967, 1988; Sturrock \& Woodburg 1967; Molodensky 1969; Nakagawa \& Raadu 1972; Nakagawa 1974; Levine 1975, 1976; Chiu \& Hilton 1977; Seehafer 1978; Sakurai 1981, 1982; Alissandrakis 1981; Low 1982, 1985; Aly 1984, 1987, 1989, 1992; Schmal et al. 1982; Wu et al. 1985; Wu et al. 1990; Gary et al. 1987; Cuperman et al. 1989a, 1989b, 1990, 1991a, 1991b, 1993; Low \& Lou 1991; Amari \& Demoulin 1992; FaubertSholl et al. 1992; Bruma \& Cuperman 1993).

Obviously, in both the potential and FFF cases, calculated chromospheric and coronal magnetic field configurations suffer from the rather limited information used for their computation: only at the bottom of the threedimensional integration domain are boundary conditions (i.e., photospheric observations) used.

Referring to the more general FFF-case (also considered in this paper), the pertaining progressive vertical extrapolation method used in the past can be summarized as follows: (a) Starting from the vector magnetograph data at the photosphere (say, $z=0$ ), use the $z$-component of the FFF-equation $\nabla \times \boldsymbol{B}=\alpha \boldsymbol{B}$ in order to calculate the function $\alpha(r)$; (b) next, by the aid of the $x$ - and $y$-components of the FFF equation, as well as of the divergence equation $\nabla \cdot \boldsymbol{B}=0$ and the $\alpha-$ function obtained at step (a), 
calculate the derivatives $\partial B_{i} / \partial_{z}(i=x, y, z)$; (c) finally, utilizing a Taylor series expansion with the first two (or three)terms retained, obtain the values $B_{i}(x, y, z)$ at a vertical (height) position $z=\mathrm{d} z$, etc. Various aspects related to the ill-posed character (in the sense of Hadamard 1923) of the progressive vertical method for the extrapolation of the photospheric magnetic field have been addressed by several authors and will not be repeated here (see, e.g., Morse \& Feshbach 1955; Tikhonov 1963; Antokhim 1968; Bakushinsky 1968; Kryanev 1973; Tikhonov \& Arsenin 1977; Low 1982, 1985; Cuperman et al. 1990). Based on the fact that many ill-posed problems do occur in science and technology these authors recommend the development and use of suitable "regularization" methods leading to satisfactory solutions.

Recently, it has been pointed out (Zhang 1993, 1994) that physical situations may exist in which, besides measurements of three-component photospheric magnetic fields, data on longitudinal (line of sight) chromospheric magnetic fields are also available; and that these two types of information can be combined in order to determine the full three-component chromospheric magnetic field. Thus, when NLTE effects are relatively small (e.g., in the case of quiet and plage regions), longitudinal chromospheric magnetograms at different wavelenghts in the blue wing of $\mathrm{H} \beta$ line are indicative of the longitudinal magnetic fields at different chromospheric altitudes. For example, using a set of eight magnetograms in the blue wind of the $\mathrm{H} \beta \lambda 4861.34 \AA$ line at wavelengths $\lambda=\lambda_{\text {core }}-[0.12 \AA+n(0.04 \AA)], n=0,1 \ldots 7$ (monochromatic images of Stoke's parameter $V$ of the $\mathrm{H} \beta$ line), and considering that the core of the $\mathrm{H} \beta$ line forms at about $1900 \mathrm{~km}$ in the solar atmosphere (Allen 1973) Zhang (1993) estimated the formation height of the wavelength $\lambda=\lambda_{\text {core }}-0.40 \AA$ to be about $1200 \mathrm{~km}$. It is anticipated that in the near future, many more $(40-50)$ multi-level longitudinal magnetic field measurements will be possible (Ai 1994a, 1994b; Fang 1994).

In this work we develop - within the FFF framework - a high order precision method for the reconstruction of chromospheric magnetic configurations. The method is based on the utilization of both types of existing magnetic field observations: (i) vector (three-component) photospheric measurements and (ii)longitudinal (along the line of sight) chromospheric measurements. The goal is to compute the full three-component magnetic field configuration at all points above active photospheric regions. A high order computational algorithm was developed and utilized for this purpose. As it will be shown, this algorithm enables one the reconstruction of the chromospheric vector magnetic field with a maximum relative error of $<10^{-3}$.

The paper is organized as follows: Sect. 2 presents the general formulation of the problem, including a discussion of the observations, the basic equations utilized and analytical solutions of FFF model equations used as a test case; Sect. 3 describes the general computational algorithm developed and its implementation; Sect. 4 presents the results of the calculation demonstrating the high performances of the algorithm; Sect. 5 elaborates on the elimination/minimization of measurements errors as well as on the fitting of the corrected data to a FFF-state; A summary is given in Sect. 6 .

\section{General formulation and basic equations}

\subsection{Vector magnetograph observations}

Modern vector magnetographs provide the following magnetic field components ${ }^{1}$ : (i) $B_{z}(x, y, 0), B_{\perp}(x, y, 0)$, $\left|B_{x}(x, y, 0)\right|$ and $\left|B_{y}(x, y, 0)\right|\left(B_{\perp}^{2}=B_{x}^{2}+B_{y}^{2}\right)$ in a horizontal plane, $D_{x y}(z=0)=L_{x} L_{y}$, at the photosphere $(z=0)$; and (ii) the longitudinal (line of sight) component $B_{z}(x, y, z)$ at a (finite) number of horizontal planes $D_{x y}$ $(z)$ situated at distances $z \simeq n \Delta z(n=1,2 \ldots)$ above the plane $D_{x y}(0)$ and parallel to it.

These observational data need some theoretical treatment before they can be used for reconstruction purposes: (a) the $180^{\circ}$ ambiguity in the observed components $B_{x}(x$, $y, 0)$ and $B_{y}(x, y, 0)$ has to be removed by the aid of some theoretical method (see, e.g., Cuperman et al. 1991b and references therein); (b) high order interpolation schemes (see, e.g., next section) have to be applied in order to obtain $B_{z}(x, y, z)$ - values at a relatively large number of intermediate horizontal planes parallel to $D_{x y}(0)$ and situated at distances $\delta z$ apart, where $\delta z(\ll \Delta z)$ represents the vertical integration step.

\subsection{Formulation of the problem}

With the magnetograph observations corrected and improved as indicated above, the problem can be formulated as follows: given (i) the transverse field components $B_{x}$ $(x, y, 0)$ and $B_{y}(x, y, 0)$, as well as the longitudinal field component $B_{z}(x, y, 0)$ in a plane $D_{x y}(z=0)$ at the photosphere and (ii) the longitudinal field component $B_{z}(x, y, z)$ at vertical distances $\delta_{z}$ apart, reconstruct the three-dimensional chromospheric magnetic field in half the space above the domain $D_{x y}(z=0)$.

\subsection{Basic equations - force free field (FFF) model}

The steady state FFF equations are

$\nabla \times \boldsymbol{B}=\alpha \boldsymbol{B}$

and

$\nabla \cdot \boldsymbol{B}=0$

Thus, Eq. (1) states that the electric current density $\boldsymbol{J}=(4 \pi)^{-1} \nabla \times \boldsymbol{B}$ is proportional to the magnetic field

1 See, e.g., Hagyard et al. (1982); Kawakami (1983); Hagyard (1985); Mein \& Rayrole (1985); Stenflo (1985); Ai (1994b). 
$\boldsymbol{B}: \boldsymbol{J}=\alpha \boldsymbol{B} / 4 \pi$. Upon taking the divergence of Eq. (1), by (2), one obtains

$\boldsymbol{B} \cdot \nabla \alpha=0$,

which indicates the constancy of $\alpha$ along individual field lines. In order of ascending complexity, Eqs. (1)-(3) may describe the cases of current-free configurations $(\alpha=0)$, linear force-free field configurations ( $\alpha=$ constant) and nonlinear force-free field configurations $[\alpha=\alpha(r)]$. The components of Eqs. (1) and (2) are:

$\frac{\partial B_{x}}{\partial z}=\alpha B_{y}+\frac{\partial B_{z}}{\partial x}$,

$\frac{\partial B_{y}}{\partial z}=-\alpha B_{x}+\frac{\partial B_{z}}{\partial y}$

$\alpha=\frac{1}{B_{z}}\left[\frac{\partial B_{y}}{\partial x}-\frac{\partial B_{y}}{\partial x}\right]$

and

$\frac{\partial B_{z}}{\partial z}=-\frac{\partial B_{x}}{\partial x}-\frac{\partial B_{y}}{\partial y}$.

Thus, if the field components at the photosphere, $B_{x}$ $(x, y ; z=0), B_{y}(x, y ; z=0)$ and $B_{z}(x, y ; z=0)$ are given (and, therefore $\alpha$ is determined) one can calculate numerically the horizontal derivatives $\partial B_{i} / \partial x_{j},(i=$ $\left.x, y, z ; x_{j}=x, y\right)$ and then, by Eqs. (4)-(7) one can obtain the vertical derivatives $\partial B_{i} / \partial z$, and proceed to the vertical integration to the height $z=\mathrm{d} z$, and so on.

\subsection{Study case}

As a study case we consider a magnetic configuration generated by electric currents satisfying the FFF condition $(\nabla \times \boldsymbol{B}=\alpha \boldsymbol{B})$ everywhere except along the line $y=0, z=-a$ (below the photosphere), where an infinite straight line current $\left(j_{l}\right)$ is flowing (Low 1982; Cuperman \& Ditkowski 1990).

The solutions of Eqs. (1) and (2) for this case are

$B_{x}=-\frac{B_{0} a}{r} \cos \phi(r)$,

$B_{y}=\frac{B_{0} a x y}{r\left[y^{2}+(z+a)^{2}\right]} \cos \phi(r)-\frac{B_{0} a(z+a)}{y^{2}+(z+a)^{2}} \sin \phi(r),($

and

$B_{z}=\frac{B_{0} a x(z+a)}{r\left[y^{2}+(z+a)^{2}\right]} \cos \phi(r)+\frac{B_{0} a y}{y^{2}+(z+a)^{2}} \sin \phi(r)$,

where

$r^{2} \equiv x^{2}+y^{2}+(z+a)^{2}$.

The free generating function $\phi(r)$ is related to the quantity $\alpha(r)$ by the equation

$\alpha(r)=-\mathrm{d} \phi(r) / \mathrm{d} r$.
In this work we use the following generating function:

$$
\phi(r)=0.2 \tanh (r /(4 a)) .
$$

Thus, in the continuation we use the values of the field components $B_{x}, B_{y}$ and $B_{z}$ (and consequently, $\alpha$ ) at the photospheric surface $(z=0)$ given by Eqs. (8)-(13) as boundary conditions and integrate the system of Eqs. (4)(7) to determine the magnetic field components in the half space $z>0$. Comparison of the results with the values given by Eqs. (8)-(12) for $z>0$ will indicate the reliability of the numerical integration procedure proposed and implemented here. $z)$ :

For convenience, we use the normalizations $(i=x, y$,

$\bar{B}_{i}=B_{i} /\left(B_{0}\right), \bar{\alpha}=\alpha a, \bar{x}=x / a, \bar{y}=y / a, \bar{z}=z / a$.

The integration limits in the $x, y$-plane are:

$-\bar{L}_{x} \leq \bar{x} \leq \bar{L}_{x}, \quad-\bar{L}_{y} \leq \bar{y} \leq \bar{L}_{y},\left|\bar{L}_{x}\right|=\left|\bar{L}_{y}\right|=b$,

$b$ being a free parameter.

\section{Computational algorithm}

The computational algorithm used in this work for the reconstruction of the three dimensional magnetic field above the photosphere consists of the several steps.

\section{1. a. Determination of the FFF-function $\alpha(r)$}

Using the observed photospheric field components $B_{i}(x$, $y, 0)(i=x, y, z)$, by the aid of Eq. (6), one calculates the non-linear FFF function $\alpha(r)$. Now, inspection of Eq. (6) reveals the existence of mathematical singularities at points at which $B_{z}=0$. While it can be shown analytically that the actual indeterminacy can be removed by $l^{\prime}$ Hôpital's techniques (see Cuperman et al. 1991b), for numerical computational purposes, some suitable methods have to be used. Thus, in our algorithm, $\alpha(r)$ is calculated at all points except in some finite width bands on both sides of the curves $B_{z}=0$; the missing $\alpha$-values are then obtained by efficient interpolation techniques. Thus, using 14-point derivative formulas (see Appendix A), $\alpha(r)$ is calculated with a maximum relative error of $\sim 10^{-8}$ ! (This maximum error occurs at the largest $r$-values considered in this work, $r \simeq 22$, where $|\alpha|$ becomes very small).

\section{2. b. Vertical extrapolation}

We perform a progressive vertical $(z)$ integration for the extrapolation of the photospheric magnetic fields within the FFF model, using as boundary conditions the field values at the photosphere. (As mentioned above, these values are simulated by the exact analytical solution of the FFF model, Eqs. (8)-(13)). 
First, from the (known) $B_{z}(x, y, z)$ - components we compute the horizontal derivatives $\partial B_{z} / \partial x$ and $\partial B_{z} / \partial y$ using the high order derivative formulas given in Appendix $A$; this is achieved with a relative accuracy of about $10^{-8}$

Second, by the aid of Eqs. (4) and (5) we obtain the vertical derivatives $\partial B_{x} / \partial z$ and $\partial B_{y} / \partial z$.

Third, using suitable 10-term extrapolation formulas developed in Appendix $B$, we obtain the sought for results - the three-component magnetic field in the half space $z>0$. The somewhat less accurate results obtained at very low vertical height $(z<10 \delta z)$ are corrected by a suitable iterative process; the maximum relative error at height $\bar{z} \equiv z / a \gtrsim 20$ is less than $10^{-3}$ !

\section{Implementation of the computational algorithm-results}

Simulation of measured photospheric field components. From the general analytical solutions, Eqs. (8)-(13) one can obtain the "photospheric" (i.e. at $z=0$ ) field components $\left|B_{x}(x, y, 0)\right|,\left|B_{y}(x, y, 0)\right|, B_{\perp}(x, y, 0)$ and $B_{z}(x, y, 0)$. For illustration, these functions are represented in Fig. 1; the "observation" domain, $D_{x y}(z=0)$ has the dimensions $\left|\bar{L}_{x}\right|=\left|\bar{L}_{y}\right|=5$ (see Eq. (15)).

Simulation of photospheric-field components, after removal of the $180^{\circ}$ ambiguity in the $B_{x}$ and $B_{y}$ components. When suitable techniques are used to remove the $180^{\circ} \mathrm{am}$ biguity (see, e.g. Cuperman et al. 1993; Li et al. 1993), one obtains the field components $B_{x}(x, y, 0)$ and $B_{y}(x, y, 0)$ shown in Figs. 2a and b. The corresponding contours of constant values are shown in Figs. 2d and e. For completness, the field component $-\left|B_{z}(x, y, 0)\right|$ is also represented in Figs. $2 c$ and f. (In Figs. 2d,e,c solid (dashed) curves indicate positive (negative) contour values; the heavy solid curves represent the contour value $B_{z}=0$ ).

Computation of the FFF-function $\alpha(r)$. Using the results illustrated in Fig. 2, by the aid of Eq. (6), one obtains the nonlinear FFF-function $\alpha(x, y)$ shown in Fig. 3a and the $\alpha(\bar{r})$-function shown in Fig. 3d (recall the definition $\left.\left[\bar{r} \equiv\left(\bar{x}^{2}+\bar{y}^{2}+(\bar{z}+1)^{2}\right)^{1 / 2}\right]\right) ;$ the corresponding relative error (N-numerical, A-analytical)

$\overline{\Delta \alpha} \equiv \frac{\Delta \alpha}{|\alpha|}=\frac{\alpha_{\mathrm{N}}-\alpha_{\mathrm{A}}}{0.5\left(\left|\alpha_{\mathrm{N}}\right|+\left|\alpha_{\mathrm{A}}\right|\right)}$

as a function of $\bar{r}$ is shown in Fig. 3e. As can be seen, the computational accuracy is exceptional: $\overline{\Delta \alpha}<10^{-8}$ ! For completness, we show in Figs. $3 b$ and $c$ the spatial dependence of the functions $\partial B_{x} / \partial y$ and $\partial B_{y} / \partial x$ which enter the expression for $\alpha$.

Electrical currents. From the $\alpha(r)$-values shown in Fig. 3a and the field components $B_{i}$, one can obtain the (normalized) FFF electrical current density components $\bar{J}_{i}=\bar{\alpha} \bar{B}_{i}(i=x, y, z)$. The computed quantities are shown in Figs. $4 a, b$ and $c$; the corresponding contours of constant values are shown in Figs. 4 d, e and c. (Here, $\left.\bar{J}_{i}=J_{i} / B_{0}\right)$.

Simulation of the longitudinal component $B_{z}(x, y, z)$. From the analytic expression, Eq. (10) one obtains discrete $B_{z}(x, y, z)$ - values in horizontal planes parallel to the observational one, at vertical distances $q \Delta z$ apart $(q=1,2, \ldots)$; these "simulated" values are indicated by circles in Fig. 5. Then, upon using a high-order interpolation method, from these values one obtains the much higher-density set of $B_{z}$-values in horizontal planes at distances $\delta z$ apart from each other, as shown by the continuous curves in Fig. 5; actually, $\delta z(\ll \Delta z)$, represents the vertical $(z)$ integration step. The top (bottom) figure represents contours of constant $B_{z}$-values in the plane $y=0(x=0)$.

Reconstruction of the magnetic field components, $B_{x}(x, y, z) \& B_{y}(x, y, z)$. Upon using Eqs. (1)-(6) with (i) "simulated" boundary conditions (at the photosphere) represented by the functions $B_{i}(x, y, 0)$ shown in Fig. 2, (ii) the non-linear FFF function $\alpha$ shown in Fig. $4 a$ a, and (iii) the "simulated" longitudinal component $B_{z}(x, y, z)$ shown in Fig. 5, by Eqs. (4)-(6) and the high order, corrective vertical extrapolation method described in Appendix $\mathrm{B}$, one obtains the final result - the three-dimensional chromospheric magnetic field. Thus, Fig. 6 illustrates the computed functions $B_{x}(x, y, z)$ and $B_{y}(x, y, z)$ at the vertical distances $\bar{z}=1,5,10$ and 20 respectively; ( $\bar{z}=0$ represents the photosphere). Figure 7 shows contours of equal values of the functions represented in Fig. 6. For completness, the spatial structure of the "measured" $B_{z}(x, y, z)$ component is also indicated. The "stretching factors" indicated on the figures $(1,1.2,1.4$ and 1.6) are used for the convenience of graphical representation.

Finally, in Fig. 8 we show contours of constant-value magnetic field components $B_{x}(x, y, z)$ and $B_{y}(x, y, z)$ in the plane $y=0$ (top) and $x=0$ (bottom); and in Fig. 9 we show the reconstructed FFF electrical current density components $\bar{J}_{i}(i=x, y, z)$ at $\bar{z}=20$ (left: $\bar{J}_{i}(x, y, z=20)$; right: corresponding contours of constant values). The average relative error $\left\langle\Delta \bar{B}_{i}\right\rangle$ and maximum relative error $\left(\Delta B_{i}\right)_{\max }$ in the computation of $B_{x}(x, y, z)$ and $B_{y}(x, y, z)$ as a function of the normalized height $\bar{z}$ are shown in Fig. 10, by solid $\left(\left\langle\Delta \bar{B}_{i}\right\rangle\right)$ and dotted $\left(\left(\Delta \bar{B}_{i}\right)_{\max }\right)$ curves. As can be seen, the computational relative accuracy is very good: $\left.\left.\left(\Delta \vec{B}_{x}\right)_{\max }\right)<510^{-5},\left(\Delta \bar{B}_{y}\right)_{\max }\right)$ $<510^{-4}$.

\section{Discussion}

In sections I-IV we formulated the problem considered in this work as follows: given the photospheric vector field $\boldsymbol{B}(\boldsymbol{x}, y, 0)$ and chromospheric line of sight component $B_{z}(x, y, z)$ satisfying FFF-conditions, reconstruct the vector field $\boldsymbol{B}(x, y, z)$ in the $3 \mathrm{D}$ space above the photosphere in which $B_{z}$ - information is available. Thus, using (i) analytical FFF solutions for $\boldsymbol{B}(x, y, 0)$ and $B_{z}(x, y, z)$ "data" 

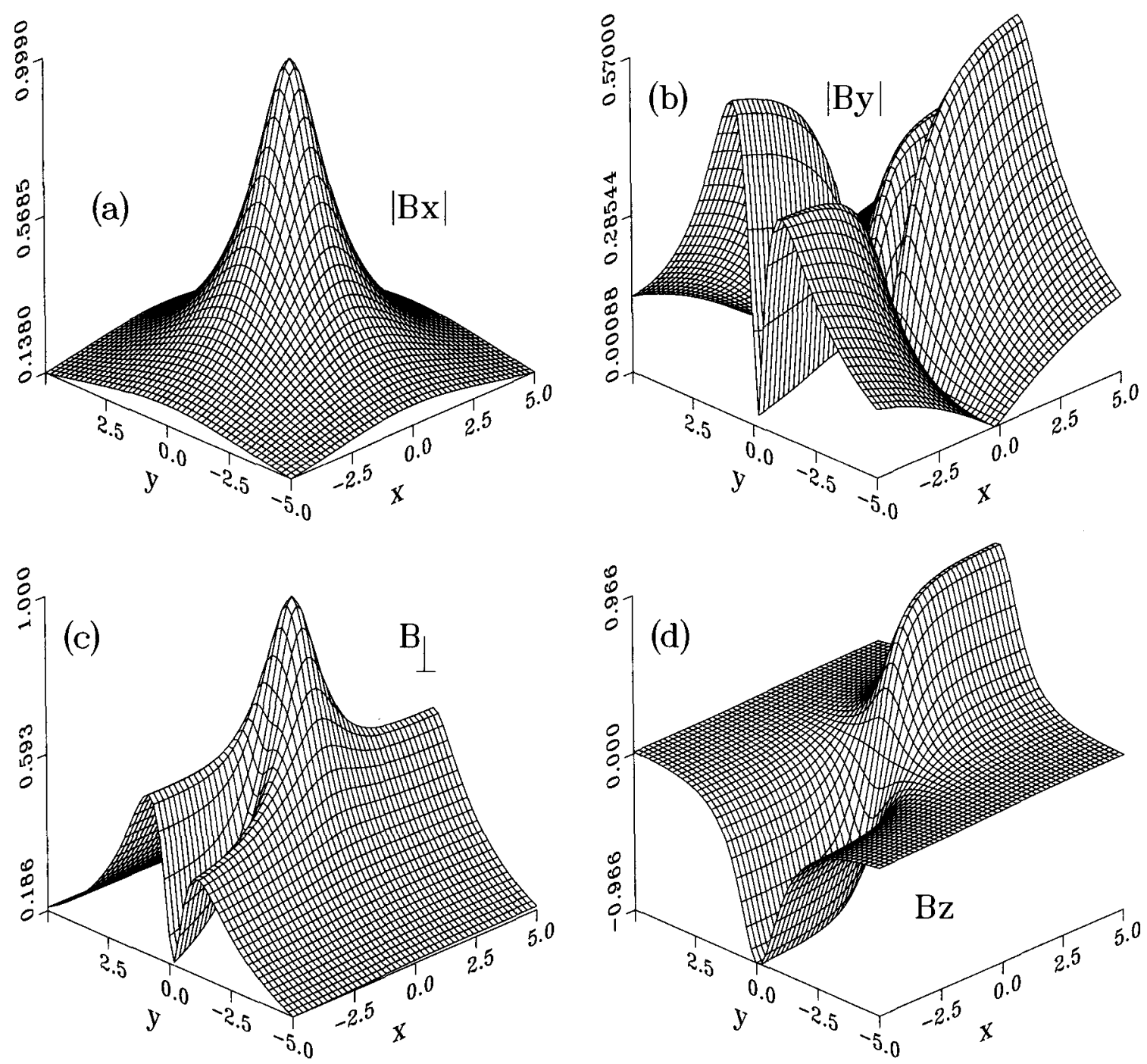

Fig. 1. Simulated photospheric $(z=0)$ observations, $\left|B_{x}(x, y, 0)\right|(\mathbf{a}),\left|B_{y}(x, y, 0)\right|(\mathbf{b}), B_{\perp}(x, y, 0)(\mathbf{c})$ and $B_{z}(x, y, 0)(\mathbf{d})$ as obtained from the analytical expressions evaluated at $z=0$. For convenience, normalized quantities are used, namely $\bar{B}_{i} \equiv B_{i} /\left(B_{0}\right),(i=x, y, z, \perp), \bar{x} \equiv x / a$ and $\bar{y} \equiv y / a$

and (ii) highly efficient computational procedures, the algorithm developed here provides results within less then $10^{-3}$ relative error.

Now, in practice, observed -rather than analytical $\boldsymbol{B}(x, y, 0)$ and $B_{z}(x, y, z)$ data have to be used. Such data are subject to the following additive sources of uncertainties: (a) projection effects, (b) $180^{\circ}$ ambiguity in the azimuth, (c) Faraday rotation of the azimuth and (d) noise in the basic data that is, in the circulary and linearly polarized intensities. Therefore, before these data can be used in the FFF - reconstruction algorithm developed in this paper, the following corrective steps have to be taken:

1. Elimination / minimization of the errors related to the uncertainty sources (a)-(d). (See, e.g. Hagyard 1985, 1988). Note that it is anticipated that space-flight magne- tograms will be characterized by polarimetric noise a factor of $10-100$ smaller than ground based systems (that is about the same as for the normal heliographic component) (see, e.g. Venkatakrishnan \& Gary 1989).

2. After the corrective procedures mentioned above are carried out, a variational modification of the "correct" data such as to fit a FFF- state is required. In this, global constraints characterizing a FFF-state have to be satisfied (see, e.g. Molodensky 1969; Aly 1984, 1989; Semel 1988).

In conclusion, we reiterate that the algorithm we developed is concerned with the basic reconstruction problem, within the framework of the FFF-model equations. It assumes that the corrective procedures indicated above have been carefully applied and that the measurement 

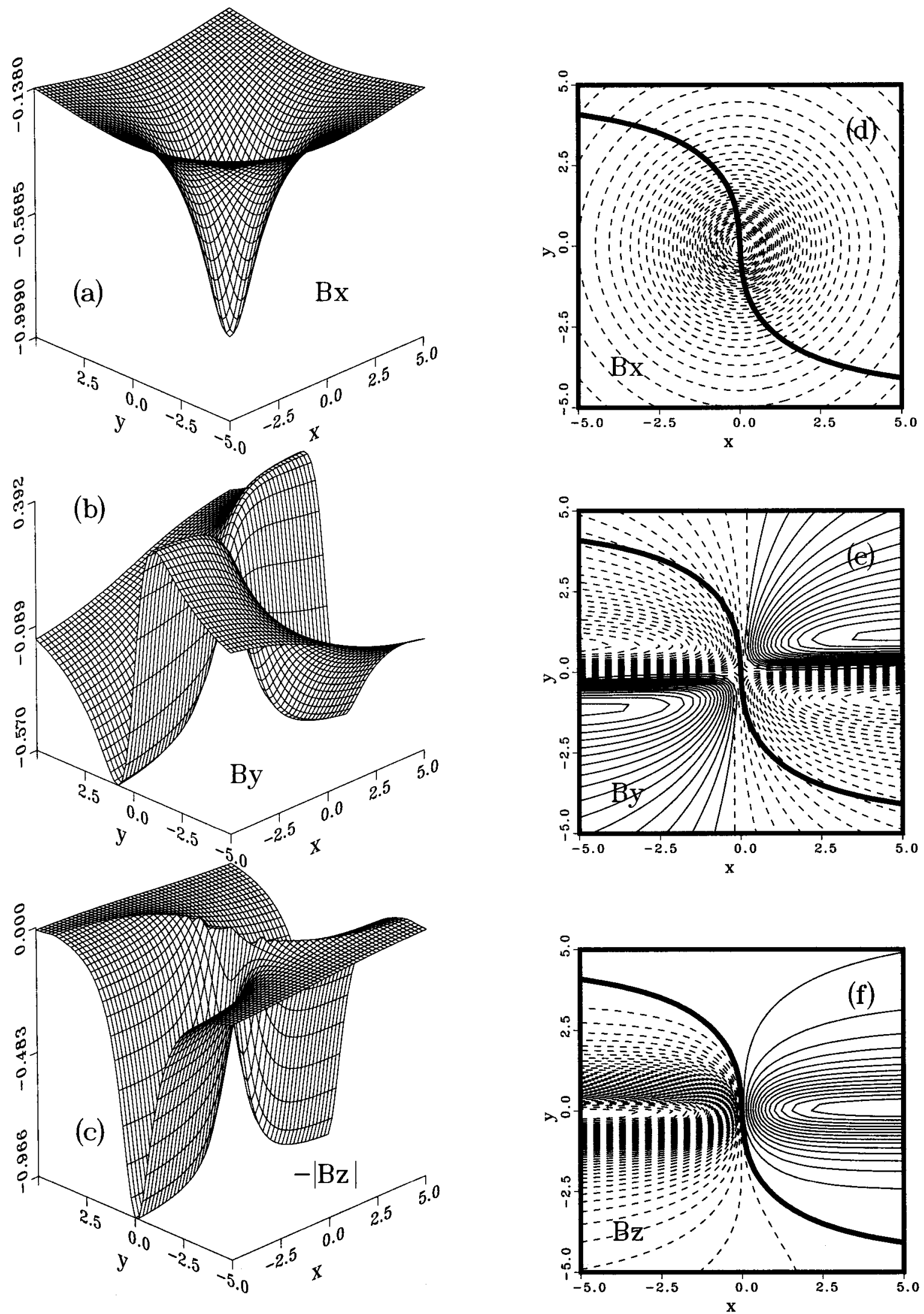

Fig. 2. Simulated photospheric $(z=0)$ observations $\bar{B}_{x}(\bar{x}, \bar{y}, 0)$ (a) and $\bar{B}_{y}(\bar{x}, \bar{y}, 0)$ (b) obtained after removal of the $180^{\circ}$ ambiguity; (d), (e): contours of constant $\bar{B}_{x}$ and $\bar{B}_{y}$-values; (c), (f) like (a), (d), for $\bar{B}_{z}(\bar{x}, \bar{y}, 0)$ 

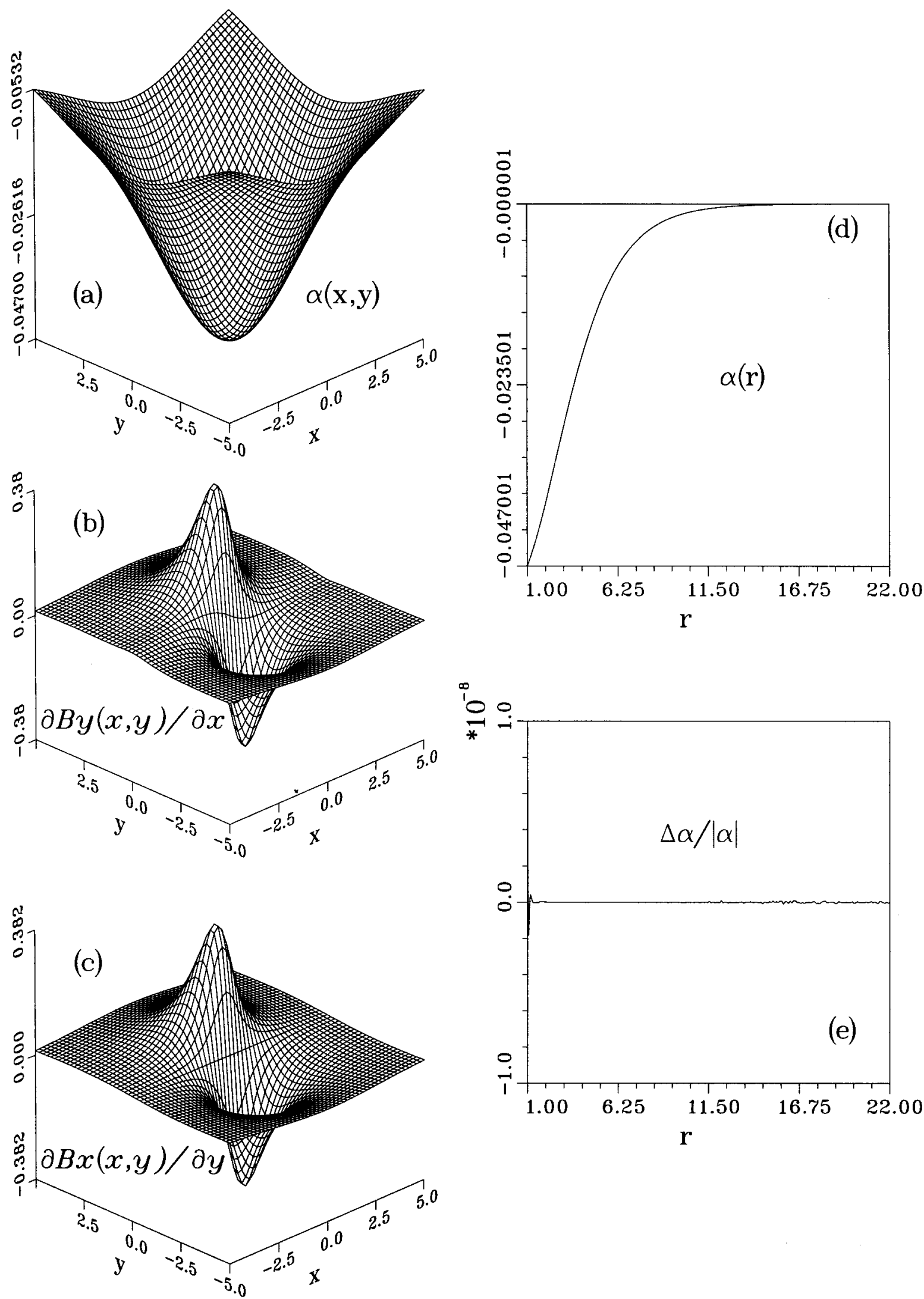

Fig. 3. (a), (d) Numerically computed non-linear FFF-function, $\alpha(\bar{x}, \bar{y}, 0)$ and $\alpha(\bar{r})$, respectively; (e) relative error $(\Delta \alpha /|\alpha|)$ as a function of $\bar{r} ;(\mathbf{b}),(\mathbf{c})$ spatial dependence of the functions $\partial B_{x} / \partial y$ and $\partial B_{y} / \partial x$ used for the calculation of the function $\alpha$ 

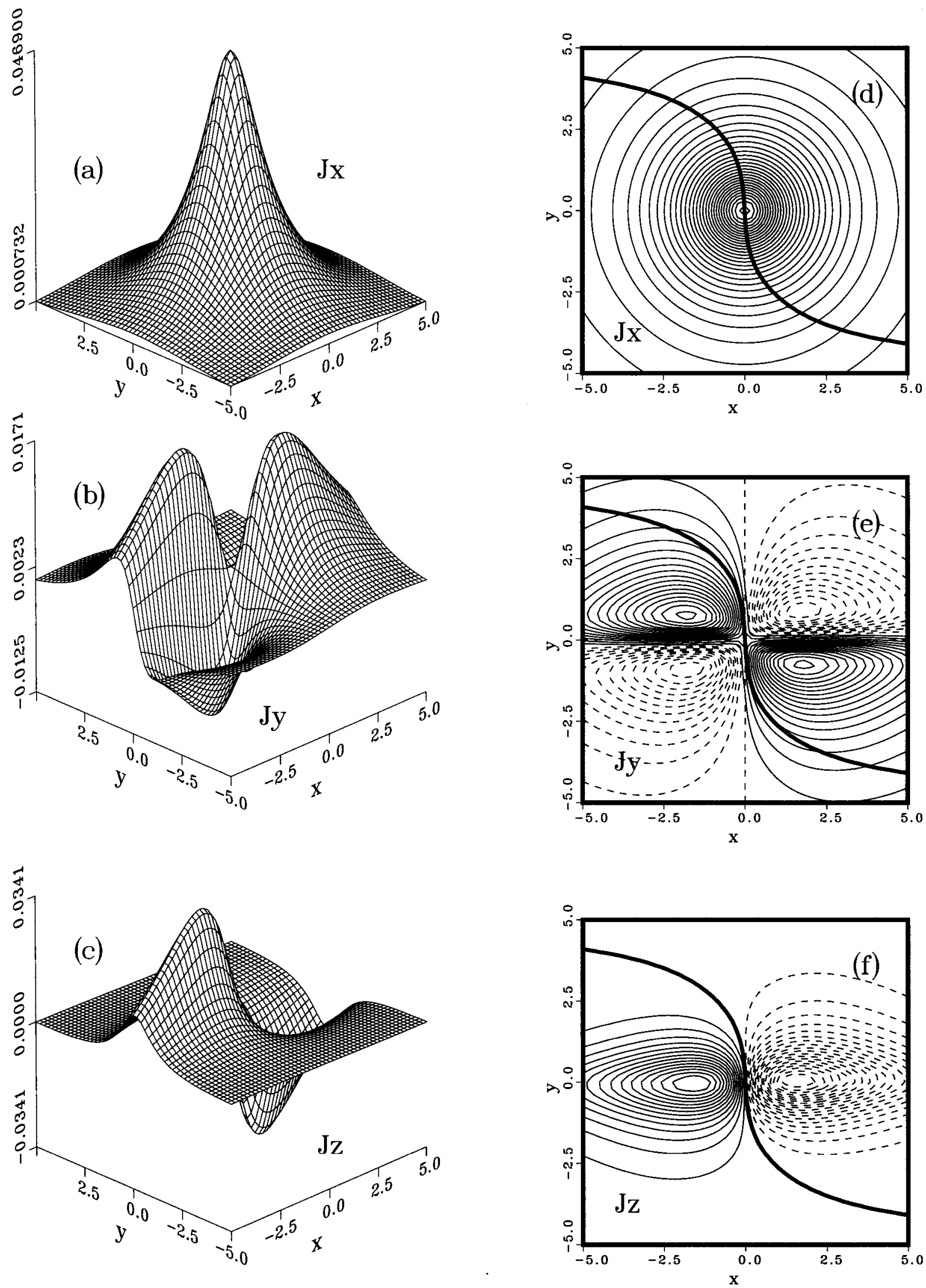

Fig. 4. (a)-(c) Photospheric electrical current densities $\bar{J}_{x}, \bar{J}_{y}$ and $\bar{J}_{z}$ based on the results shown in Fig. 2 and Fig. 3; (d)-(f) corresponding contours of constant current values 

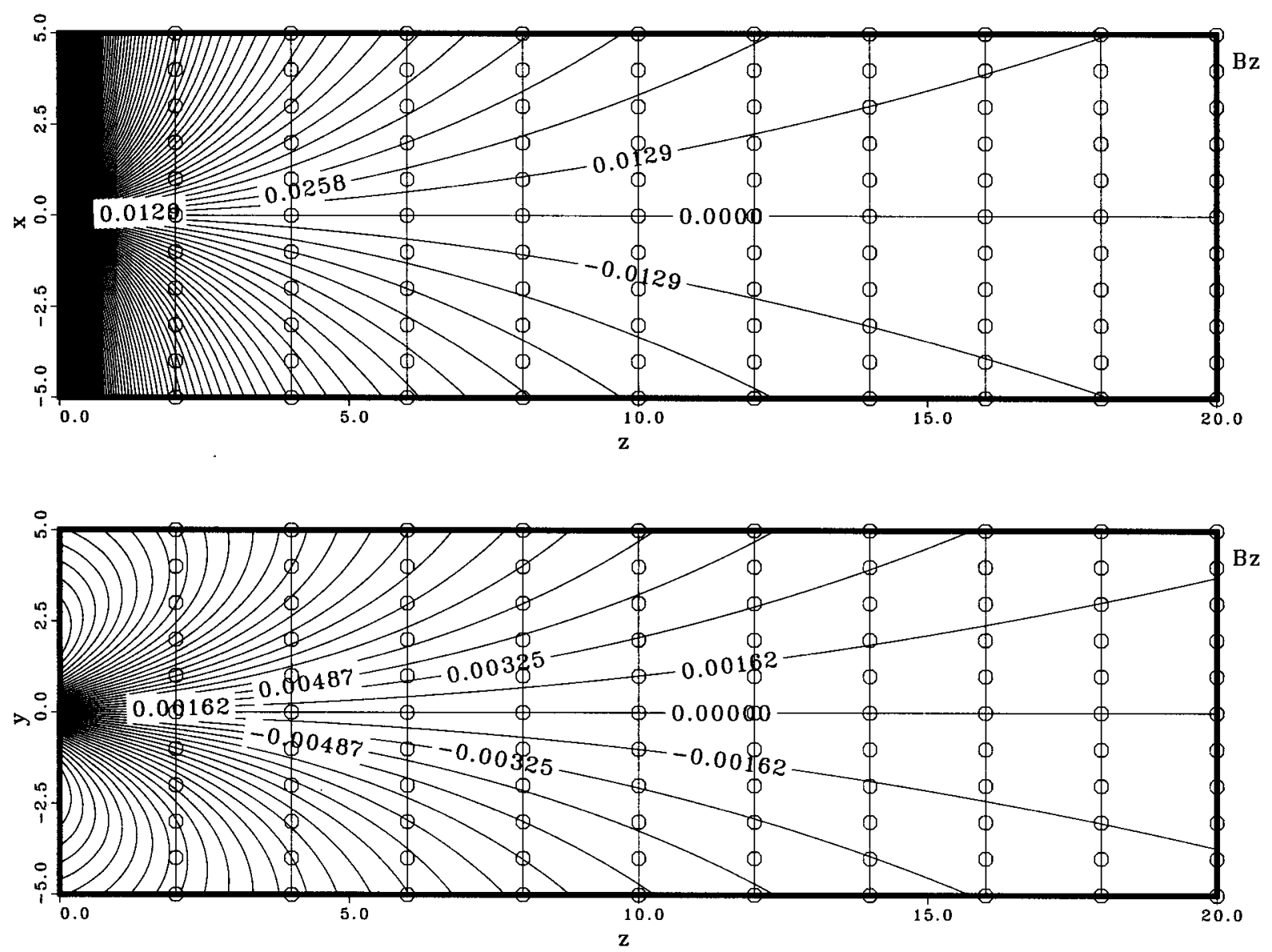

Fig. 5. Circles: "Simulated observations" of $B_{z}(x, y, z)$ - contours as obtained from the analytical expression; solid curves: interpolated values obtained upon using high order interpolation techniques. Top (bottom): $y=0(x=0)$ planes

errors as well as the deviations of the data from a FFFstate have been reduced to an insignificant level.

\section{Summary}

We have developed and implemented a computational algorithm for the reconstruction of the vector - chromospheric magnetic fields within the framework of the non-linear FFF theoretical model. This algorithm represents the extension, modification and improvement of previously proposed progressive vertical extrapolations (see, e.g. Wu et al. 1985; Cuperman et al. 1990) in the following sense:

1. In the calculation of the non-linear FFF-function $\alpha$ (by the aid of Eq. (6)), the derivatives $\partial B_{y} / \partial x$ and $\partial B_{x} / \partial y$ are computed by the aid of 14 -term formulas, rather than by $2-3$ term formulas as previously used. Moreover, 14-versions of the 14-term formulas are used at various points, in order to ensure about equal minimum computational error. The maximum relative error in the computation of these derivatives is smaller than
$10^{-10}$. As a consequence, $\alpha$ is computed with a maximum relative error smaller than $10^{-8}$ (see Fig. 3).

2. The same 14-term formula (and its 14 versions) is applied for the calculation of the derivatives $\partial B_{z} / \partial x$ and $\partial B_{z} / \partial y$ which enter the expressions of $\partial B_{x} / \partial z$ and $\partial B_{y} / \partial z$, Eqs. (4) and (5), respectively. Consequently, the same high computational precision is obtained also for this purpose.

3. At points where $B_{z}=0$, to avoid inherent "mathematical" discontinuities, rather than calculating $\alpha(r)$ by the aid of Eq. (6) in conjunction with some smoothing techniques, a suitable, different approach is used. The same holds at points where $B_{y}=0$ or $B_{x}=0$, including the case in which $B_{z}=B_{y}=0$. Thus, at all points in the range $1 \leq \bar{r} \lesssim 22$, the maximum relative error in the computed function $\alpha$ is smaller than $10^{-8}$ (See Fig. 3).

4. The progressive vertical $(z)$ extrapolation is based on a "moving" 10-term formula, including information from ten consecutive grid points, $q=0,1,2, \ldots, 9$. Thus, the extrapolated value $B_{i}(x, y, q)$ is expressed in terms of its derivatives at grid points $q=0,1,2, \ldots, 9$ as well as the 

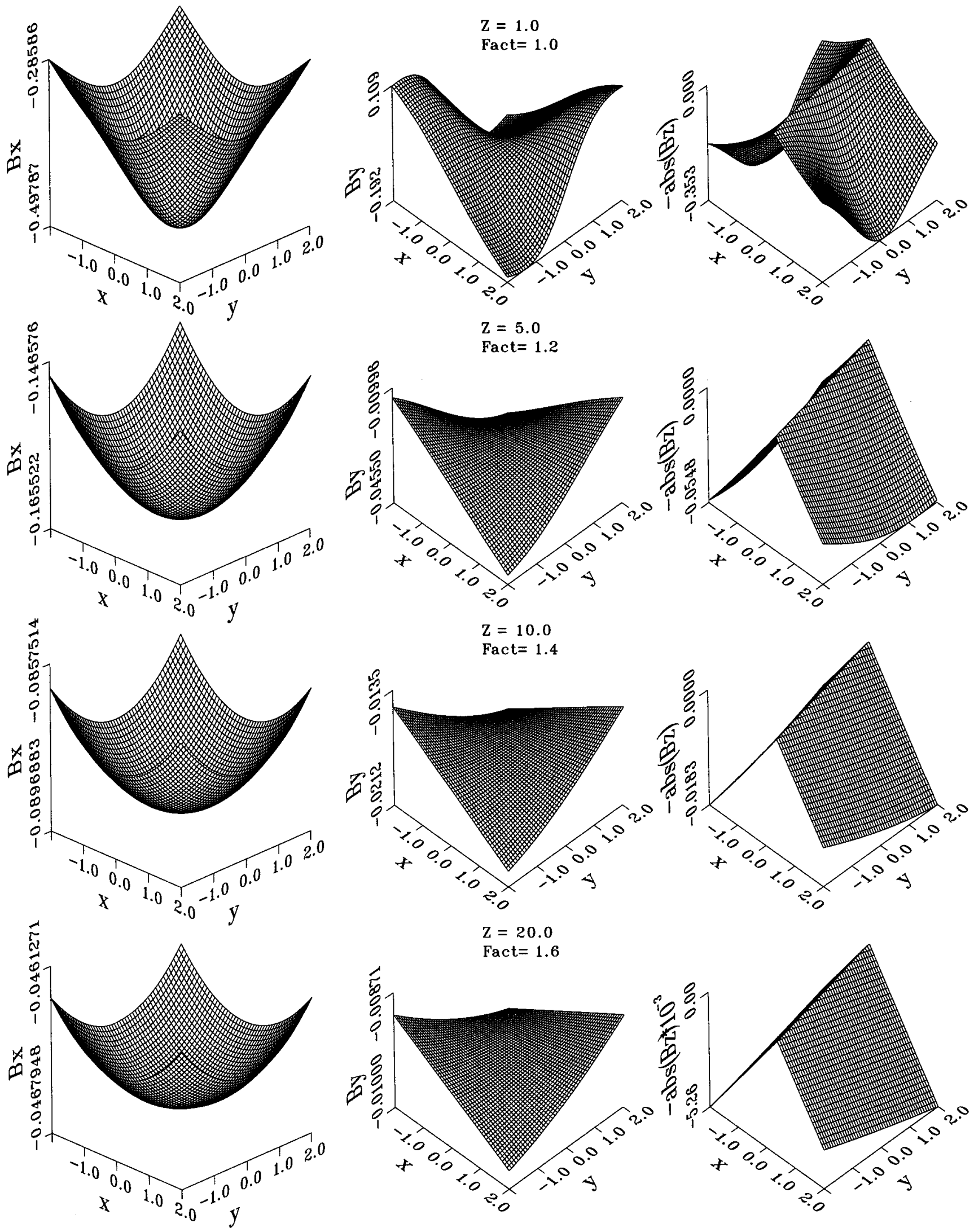

Fig. 6. Reconstructed magnetic field components $\bar{B}_{x}(\bar{x}, \bar{y}, \bar{z})$ (left column) and $\bar{B}_{y}(\bar{x}, \bar{y}, \bar{z})$ (middle column) at several height values $\bar{z}$, as indicated; for comparison, the corresponding $\bar{B}_{z}(\bar{x}, \bar{y}, \bar{z})$ - component is shown in the right column. (Actually, to emphasize the neutral line, $\bar{B}_{z}=0$, we represent the quantity $-\left|\bar{B}_{z}(\bar{x}, \bar{y}, \bar{z})\right|$, rather than just $\left.\bar{B}_{z}(\bar{x}, \bar{y}, \bar{z})\right)$ 
$\mathrm{Z}=1.0 \quad$ Fact $=1.0$
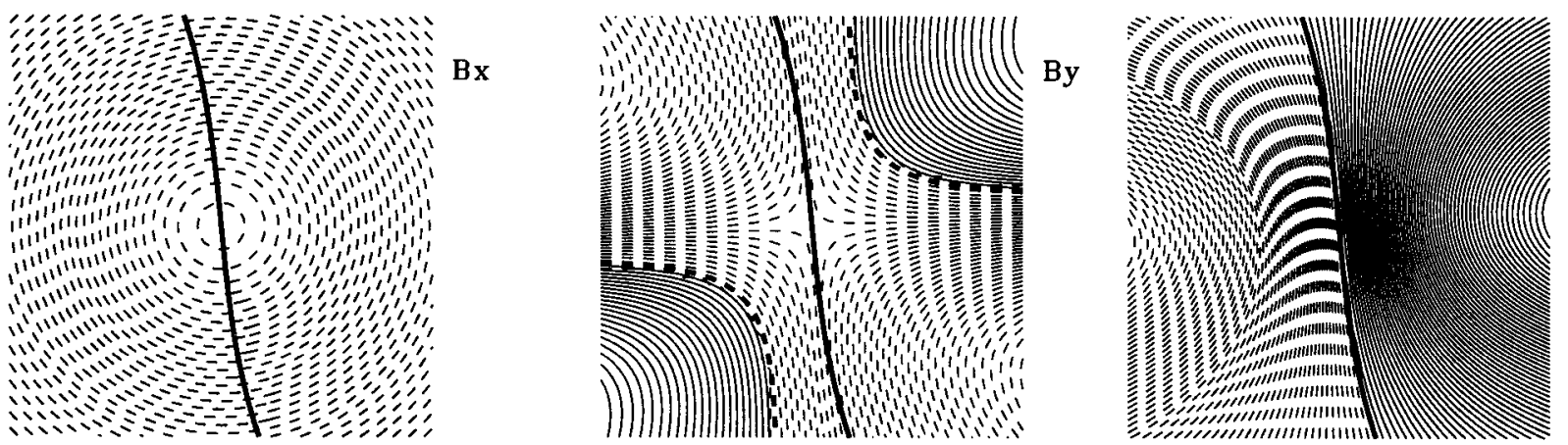

$\mathrm{Bz}$

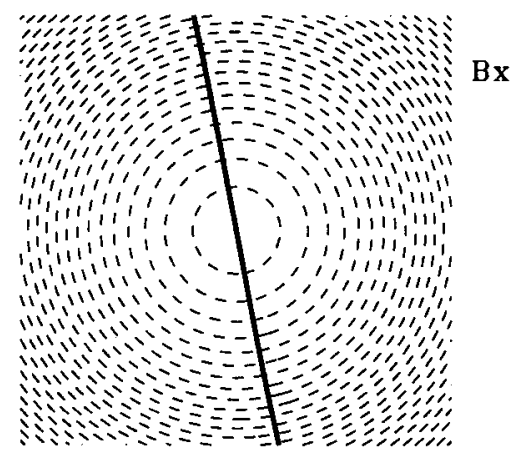

\section{$\mathrm{Z}=5.0 \quad$ Fact $=1.2$}

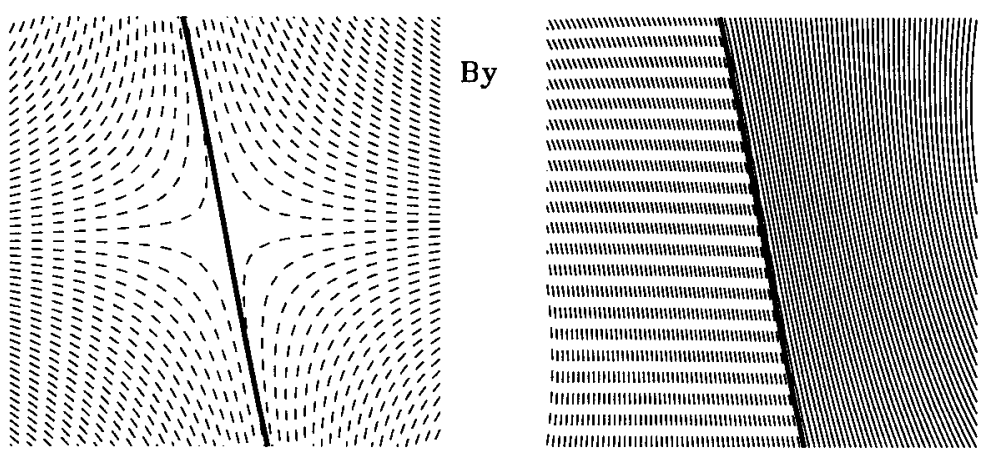

$\mathrm{Bz}$

$$
\mathrm{Z}=10.0 \quad \text { Fact }=1.4
$$
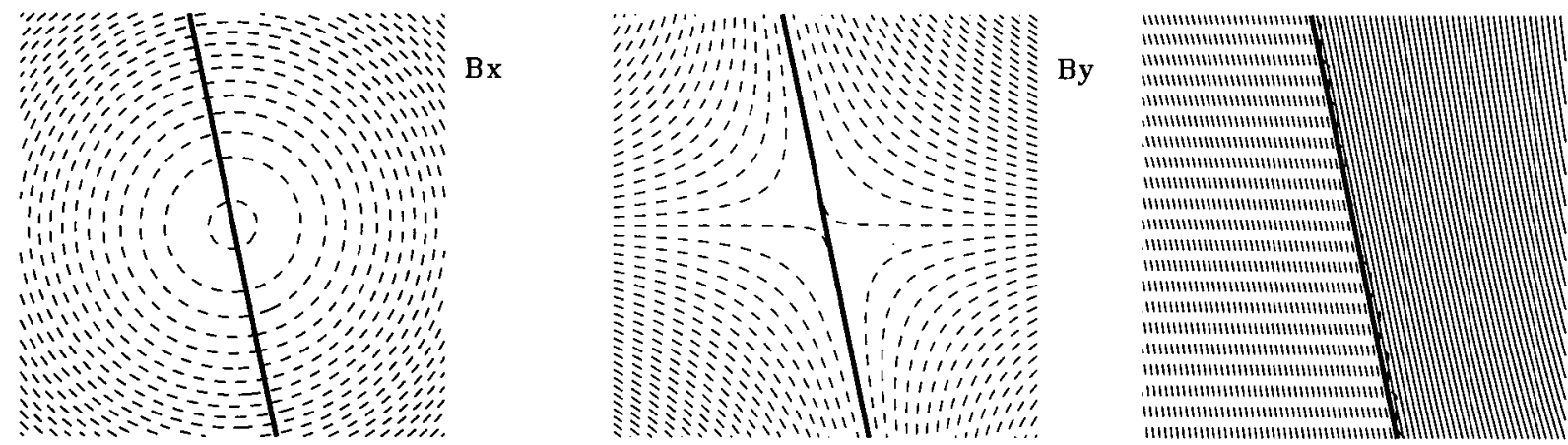

$\mathrm{Bz}$
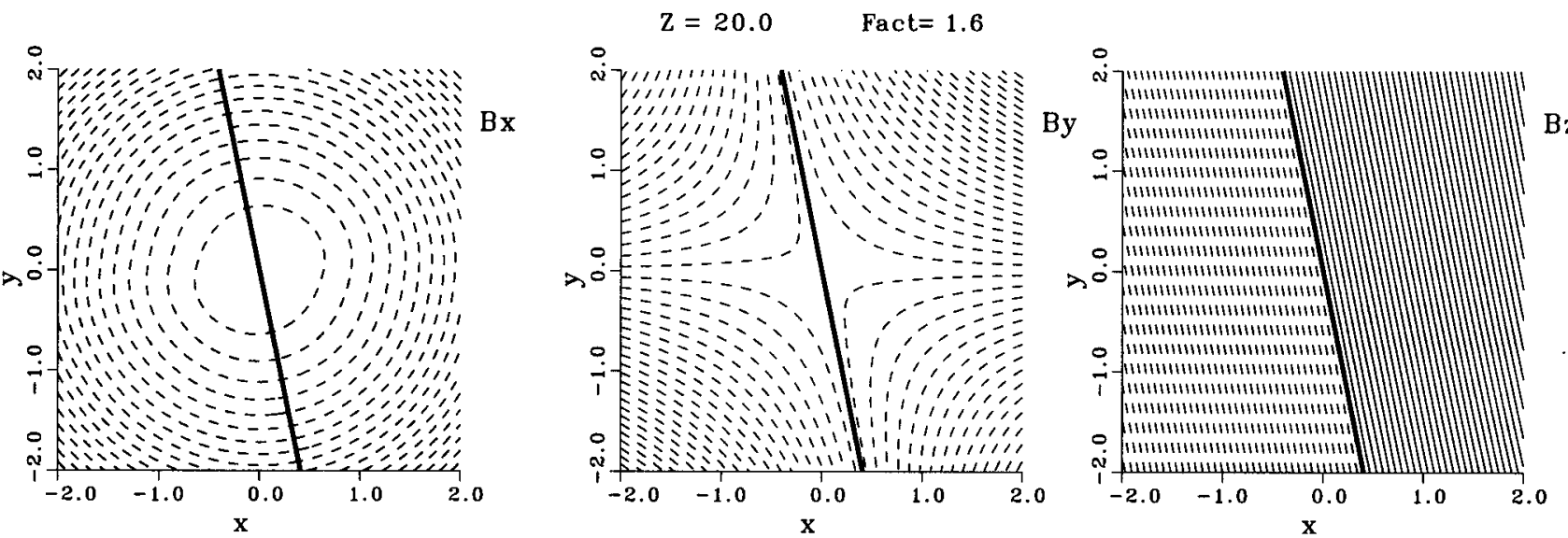

Fig. 7. Contours of equal values of the functions represented in Fig. 6 

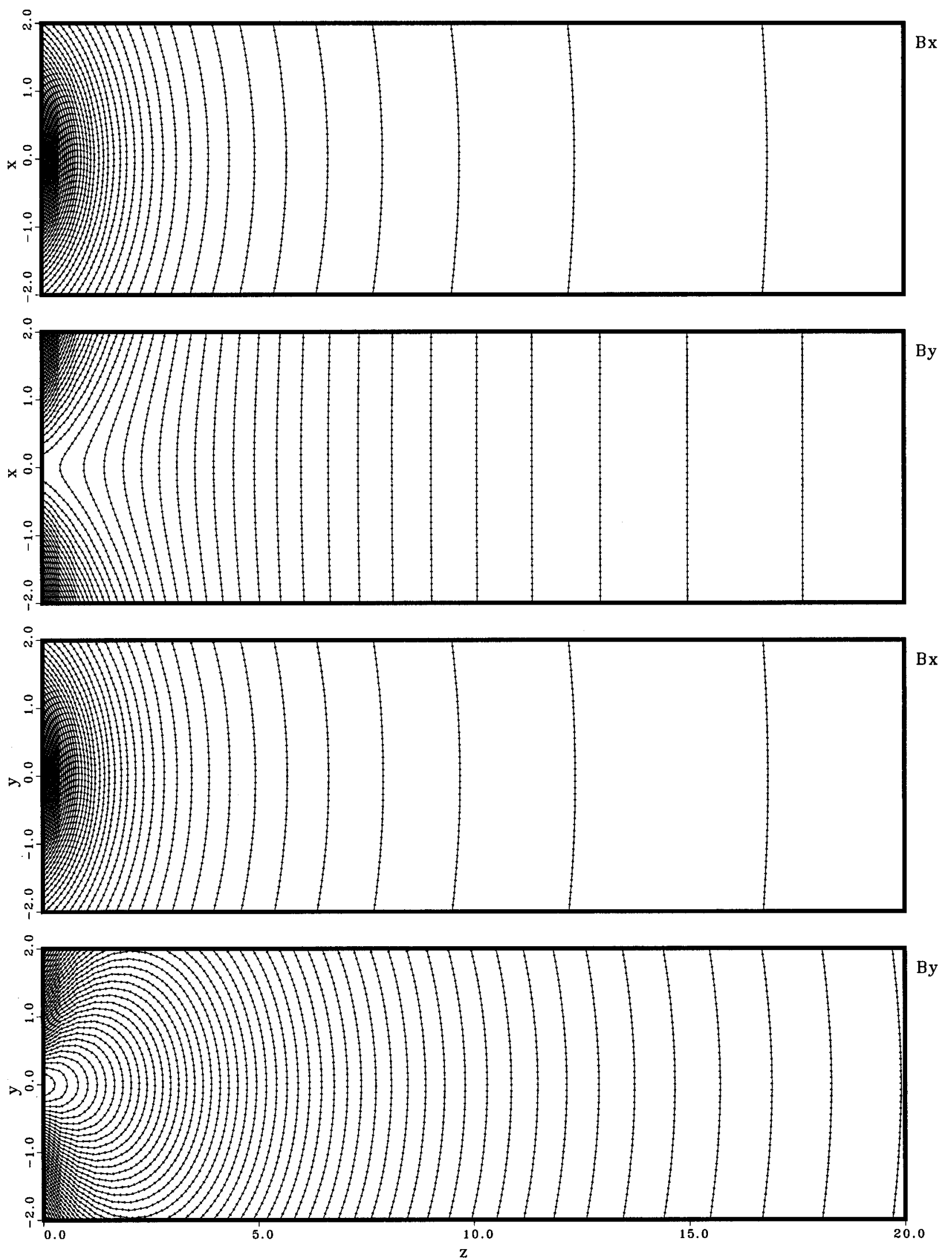

Fig. 8. Contours of constant magnetic field values $\bar{B}_{x}(\bar{x}, \bar{y}, \bar{z})$ and $\bar{B}_{y}(\bar{x}, \bar{y}, \bar{z})$ in the plane $y=0$ (top) and $x=0$ (bottom) 

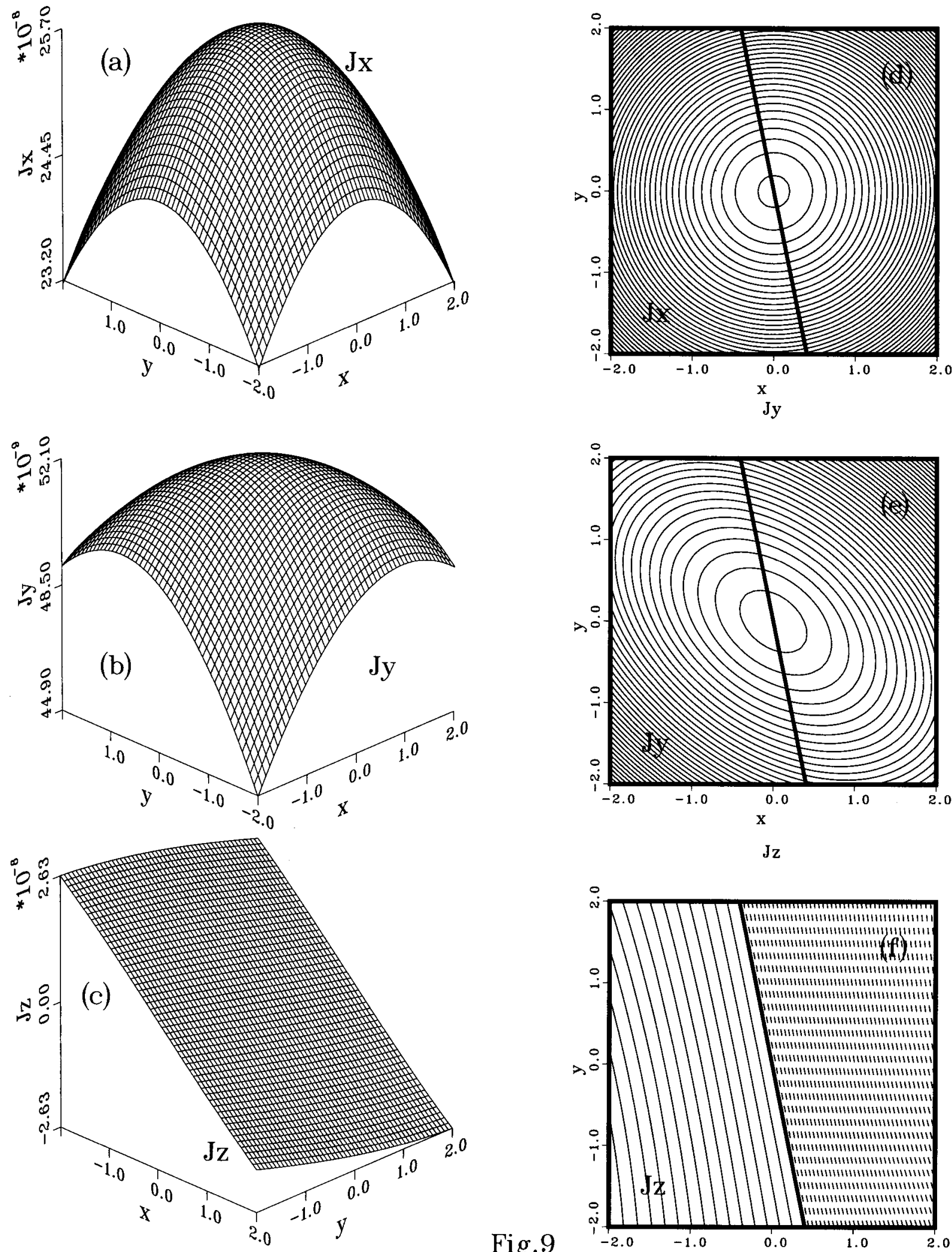

$\mathbf{J z}$

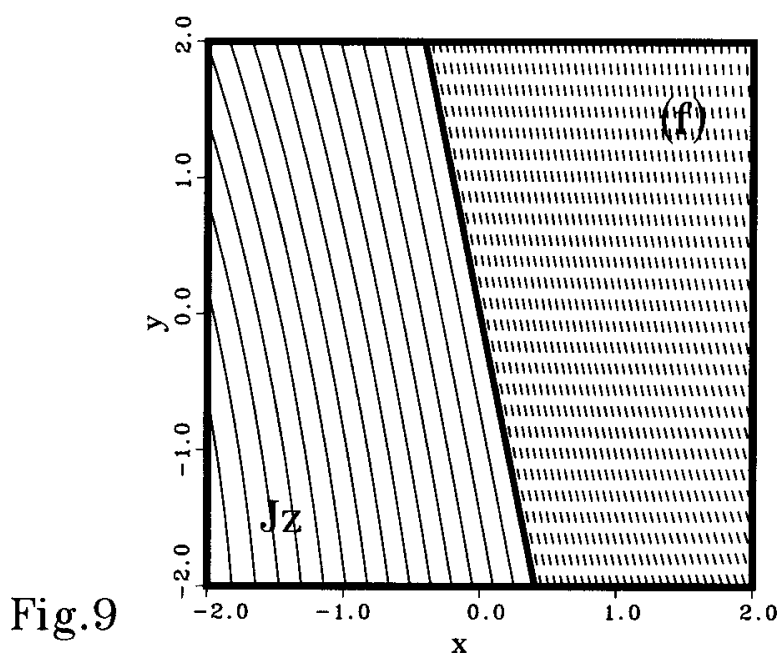

Fig. 9. Left: Reconstructed FFF electrical current densities $\bar{J}_{x}(\bar{x}, \bar{y}, \bar{z}), \bar{J}_{y}(\bar{x}, \bar{y}, \bar{z})$ and $\bar{J}_{z}(\bar{x}, \bar{y}, \bar{z})$ at several height values $\bar{z}$, as indicated; right: corresponding contours of constant current values 
value of the function itself at the point $q=0$; the various derivatives are calculated according to Eqs. (4) and (5), with $\partial B_{z} / \partial x, \partial B_{z} / \partial y$ and $\alpha$ obtained as indicated above.

5. A special correction is applied to the extrapolation results obtained for the first few grid points above the photosphere (especially at the first point, $\bar{z}=\delta \bar{z}$ ) because in these cases the information required by the "ten-term formula" is not available.

6. As a result ot the computational algorithm summarized above, an exceptional good extrapolation accuracy is obtained: at $\bar{z}=20(\equiv 500 \delta \bar{z})$, the maximum relative error in the extrapolated vector magnetic field component $B_{x}\left(B_{y}\right)$ is smaller than $10^{-4}\left(10^{-3}\right)$.
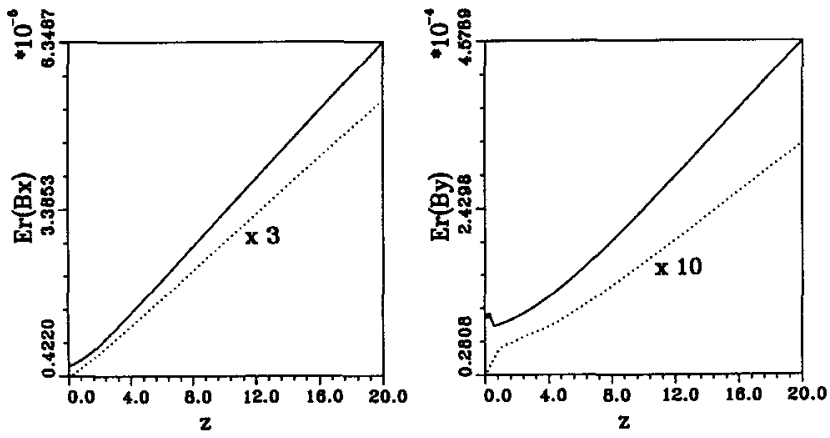

Fig. 10. Average relative error $\left\langle\Delta \bar{B}_{i}\right\rangle$ (solid curve) and maximum relative error (dotted curve) in the reconstruction of the magnetic field components $\bar{B}_{x}(\bar{x}, \bar{y}, \bar{z})$ and $\bar{B}_{y}(\bar{x}, \bar{y}, \bar{z})$ as a function of the normalized height, $\bar{z}$

Acknowledgements. The authors are grateful to G. Ai, J.J. Aly, P. Demoulin, C. Fang, J.C. Henoux, B. Leroy, J. Rayrole, T. Sakurai, M. Semel and H. Zhang for valuable discussions or/and for a critically reading of the manuscript.

\section{Appendices}

\section{A. Horizontal $(x, y)$ derivatives}

Following the computational algorithm developed by Bruma \& Cuperman (1996) for the calculation of horizontal derivatives in problems of the type studied here, we use a "flexible" fourteen-grid-point formulation designed to achieve optimal accuracy.

1 . Denote by ${ }^{2}\left(x_{i, 0}, x_{i, 1}, \ldots, x_{i, l}, \ldots x_{i, s}\right)$ the coordinates of a set of equidistant grid points along the $x$-axis and by $F\left(x_{i, 0}\right), F\left(x_{i, 1}\right), \ldots, F\left(x_{i, l}\right), \ldots F\left(x_{i, s}\right)$ the values of the function $F$ at the corresponding grid points; here $F$ stands for either $B_{x}(x, y, z), B_{y}(x, y, z)$ or $B_{z}(x, y, z)$. Also, use the notation $P 1\left(x_{i, l}\right) \equiv(\partial F / \partial x)_{x_{i, l}} . \Delta x$, where $\Delta x$ represents the equidistant grid size.

2 The same holds for the $y$-direction.
2. Concerning the meaning of subscripts used: (a) the subscripts $l=0,1,2, \ldots, s,(s=14)$ define a "moving" set of fifteen grid-point numbers; (b) the subscripts $(i=1,2, \ldots)$ indicate the order of the moving-set along the $x$-axis, starting at $x_{\min }$ (where $i=1$ ) and ending at $x_{\max }$.

3. At a point $x_{i, l} \equiv x_{k}$, the optimal value of the derivative $P 1\left(x_{k}\right)$ is obtained through a systematic investigation leading to the minimization of the relative error involved. This procedure leads to one of the following two possibilities: (a) use of a symmetric fourteen-point formula, with seven points on each side of the point $x_{k}$ or, (b) use of a non-symmetric variable-number-of-terms-formula $(n \lesssim 14)$ at the left or at the right of $x_{k}$.

For illustration, in the case (a), one has $\left(x_{k}=0,1, \ldots, 14\right)$

$$
\begin{aligned}
P 1(7)= & (15 F(14)-245 F(13)+1911 F(12) \\
& -9555 F(11)+35035 F(10)-105105 F(9) \\
& +315315 F(8)-315315 F(6)+105105 F(5) \\
& -35035 F(4)+9555 F(3)-1911 F(2) \\
& +245 F(1)-15 F(0)) / 360360 .
\end{aligned}
$$

For the case (b)

$$
\begin{aligned}
P 1(0)= & (-25740 F(14)+388080 F(13) \\
& -2732730 F(12)+11924640 F(11) \\
& -36072036 F(10)+80160080 F(9) \\
& -135270135 F(8)+176679360 F(7) \\
& -180360180 F(6)+144288144 F(5) \\
& -90180090 F(4)+43723680 F(3) \\
& -16396380 F(2)+5045040 F(1) \\
& -1171733 F(0)) / 360360 .
\end{aligned}
$$

A complete description on the general algorithm, including the criteria for the selection of the formulas for the horizontal derivations is given in Bruma \& Cuperman (1996).

\section{The vertical $(z)$ integration}

The analysis by Bruma \& Cuperman (1996) clearly indicates that the accuracy of the vertical $(z)$ integration in problems of the type considered here, can be significantly improved upon formulating the problem in terms of the value of the function to be integrated $(F)$ at $z=0$ and its derivatives at (equidistant) grid points above $z=0$. We here present some final results obtained in the reference indicated above.

Denote by $\left(z_{j, 0}, z_{j, 1}, . ., z_{j, k}, \ldots z_{j, n}\right)$ the coordinates of a set of equidistant grid points along the $z$-axis and by $F\left(z_{j, 0}\right), F\left(z_{j, 1}\right), \ldots . . F\left(z_{j, k}\right) \ldots . . F\left(z_{j, n}\right)$ the values of the function $F$ at the corresponding grid points. Let $n \lesssim 9$ 
and $j \lesssim 500$; then, about 500 grid points are involved. The first "moving" set is labeled $j=0$ and starts at the photosphere, $z_{0,0} \equiv 0$. (Notice that here $F$ represents either $B_{x}(x, y, z)$ or $\left.B_{y}(x, y, z)\right)$.

By the aid of the functions $F\left(z_{j, k}\right)(z=0,1, . ., n)$ it is possible to calculate their first order derivatives, $P 1\left(z_{z, k}\right)^{3}$; then, in the resulting system of relationships, we eliminate the functions $F\left(z_{j, 1}\right), \ldots \ldots F\left(z_{j, n-1}\right)$, as well as the derivative $P 1\left(z_{j, n}\right)$ to obtain a relation of the form

$F\left(z_{j, n}\right)=f\left\{F\left(z_{j, 0}\right), P 1\left(z_{j, 0}\right), \ldots, P 1\left(z_{j, n-1}\right)\right\}$.

Thus, at a grid point $z_{j, n}$ we have a ten-grid-point-formula for the function $F$ in terms of the $F$-value at $z_{j, 0}$ and its derivatives at all other points. In particular, starting at the photosphere where $z_{j, 0} \equiv z_{0,0}=0$, the above symbolic formula reads

$F(n)=f\{F(0) ; P 1(1), \ldots, P 1(9)\}$

The explicit relation (20) is obtained by the aid of the REDUCE-package and it reads

$$
\begin{aligned}
F(9)= & (44800 F(0)-25713 P 1(0) \\
& +44874 P 1(1)-467694 P 1(2) \\
& +992898 P 1(3)-1645920 P 1(4) \\
& +1593918 P 1(5)-1166994 \\
& P 1(6)+457974 P 1(7) \\
& -186543 P 1(8)) / 44800 .
\end{aligned}
$$

At this point the following remark is in order: at grid points $z_{0, k<n}$, some simpler integration formulas can be used, namely:

$$
\begin{aligned}
F(1)= & F(0)-P 1(0) \\
F(2)= & F(0)-2 P 1(1) \\
F(3)= & (4 F(0)-3 P 1(0)-9 P 1(2)) / 4 \\
F(4)= & (3 F(0)-8 P 1(1)+4 P 1(2)-8 P 1(3)) / 3 \\
F(5)= & (144 F(0)-95 P 1(0)+50 P 1(1) \\
& -600 P 1(2)+350 P 1(3)-425 P 1(4)) / 144 \\
& -78 P 1(3)+42 P 1(4)-33 P 1(5)) / 10 \\
F(6)= & (10 F(0)-33 P 1(1)+42 P 1(2) \\
& -59829 P 1(2)+81536 P 1(3) \\
& -102459 P 1(4)+50568 P 1(5) \\
& -30919 P 1(6)) / 8640
\end{aligned}
$$

3 We use the notation $\partial F / \partial x \equiv P 1 / \Delta z$, where $\Delta z$ represents the (equidistant) grid size.

$$
\begin{aligned}
F(8)= & (945 F(0)-3680 P 1(1)+7632 P 1(2) \\
& -17568 P 1(3)+19672 P 1(4) \\
& -17568 P 1(5)+7632 P 1(6) \\
& -3680 P 1(7)) / 945 .
\end{aligned}
$$

As proposed and implemented by Bruma \& Cuperman (1996), utilization of an appropriate iterative procedure leads to improvement of the zero-order results obtained at very low levels $(1 \lesssim n<9)$.

\section{References}

Ai G., 1994a (private communication)

Ai G., 1994b, Space Solar Telescope, Beijing Astronomical Observatory, Beijing 100080, R.P. China

Alissandrakis E.E., 1981, A\&A 100, 197

Allen C.W., 1973, Astrophys. Quant., 3d ed. The Athlone Press, London

Amari T., Demoulin P., 1992, in Méthodes de Détermination des Champs Magnétiques Solaires et Stellaires, FaurobertSchool M., Frish H., Mein N. (eds.). Observatoire de Paris Antokhim Yu.T., 1968, Differentisial'nyye Uravneniya 4, 9

Aly J.J., 1984, ApJ 283, 349

Aly J.J., 1987, Interstellar Magnetic Fields, Beck R., Grave R. (eds.). Springer, Berlin, Heidelberg, New York, p. 240

Aly J.J., 1989, Solar. Phys. 120, 19

Aly J.J., 1992, Solar. Phys. 138, 133

Bakushinskiy A.B., 1968, Dokl. Akad. Nauk SSSR 183, 1

Bruma C., Cuperman S., 1993, A\&A 278, 589

Bruma C., Cuperman S., 1996, Report TAUP - 232296, School of Physics and Astronomy, Tel Aviv University, Israel

Chiu Y.T., Hilton H.A., 1977, ApJ 212, 873

Cuperman S., Ofman L., Semel M., 1989a, A\&A 227, 227

Cuperman S., Ofman L., Semel M., 1989b, A\&A 216, 265

Cuperman S., Ofman L., Semel M., 1990, A\&A 230, 193

Cuperman S., Ditkowski A., 1991a, A\&A 241, 646

Cuperman S., Demoulin P., Semel M. 1991b, A\&A 245, 288

Cuperman S., Li J., Semel M., 1993, A\&A 278, 279

Demoulin P., Cuperman S., Semel M., 1992, A\&A 263, 351

Faubert-Scholl M., Frisch H., Mein H., 1992, Méthodes de Détermination des Champs Magnétiques Solaires et Stellaires, Observatoire de Paris

Fang C., 1994 (private communication)

Gary G.A., Moore R.L., Hagyard M.J., Haiasch B.M., 1987, ApJ 314, 782

Gary G.A., 1988, Linear force-free magnetic fields for solar Extrapolation and Interpretation, Space Science Laboratory, Marshall Space Flight Center, Preprint Series No. 88-144

Grad H., Rubin H., 1958, in Proc. 2nd Intern. Conf. Peaceful Uses of Atomic Energy, Vol. 31, Geneva, United Nations, p. 140

Hadamard J., 1923, Lectures on Cauchy's Problem in Linear Partial Differential Equations. Yale University Press, Yale

Hagyard M.J., 1985, Measurements of Solar Vector Magnetic Fields, NASA CP-2374, Marshall Space Flight Center Workshop

Hagyard M.J., Cumings N.P., West E.A., Smith J.E., 1982, Solar Phys. 80, 33

Hagyard M.J., 1988, Solar Phys. 115, 107 
Kawakami H., 1983, PASJ 35, 459

Krall K.R., Smith J.B. Jr., Hagyard M.J., West E.A., Cumings N.P., 1982, Solar Phys. 79, 1982

Kryanev A.V., 1973, Dokl. Akad. Nauk SSSR 210, 1

Levine R.H., 1975, Solar Phys. 44, 365

Levine R.H., 1976, Solar Phys. 46, 159

Li J., Cuperman S., Semel M., 1993, A\&A 279, 214

Low B.C., 1982, Solar Phys. 77, 43

Low B.C., 1982, Measurements of Solar Vector Magnetic

Fields. In: Hagyard M.J. (ed.). NASA CP-2374, Marshall

Space Flight Center Workshop, 49

Low B.C., Lou Y.Q., 1991, ApJ 352, 343

Mein P., Rayrole J., 1985, Vis. Astron. 28, 567

Molodenski M.M., 1969, Soviet. Astr. - A.J. 10, 585

Molodenski M.M., 1974, Solar Phys. 39, 393

Morse P.M., Feshbach H., 1955, Methods in Theoretical Physics. McGraw Hill, New York

Nakagawa Y., 1974, ApJ 190, 437

Nakagawa Y., Raadu M.A., 1972, Solar Phys. 25, 217

Sakurai T., 1981, Solar Phys. 69, 243

Sakurai T., 1982, Solar Phys. 67, 301

Sakurai T., 1989, Space Sci. Rev. 51, 11
Schmal E.J., Kundu M.R., Strong K.T., Bentley R.D., Smith J.B. Jr., Krall K.R., 1982, Solar Phys. 80, 233

Schmidt H.U., 1964, in Physics of Solar Flares, Hess W.N. (ed.). NASA SP-50, 107

Seehafer N., 1978, Solar Phys. 58, 215

Semel M., 1967, Ann. Astrophys. 30, 513

Semel M., 1988, A\&A 198, 293

Stenflo J.O., 1985, Vis. Astron. 28, 567

Sturrock P.A., Woodbury E.T., 1967, in Plasma Astrophysics, Sturrock P.A. (ed.). Academic Press, New York, p. 155

Tikhonov A.N., 1963, Dokl. Nauk SSSR 153, 1

Tikhonov A.N., Arsenin V.Y., 1977, Solutions of ill-posed problems. Wiley, New York

Venkatakrishnan P., Gary G.A., 1989, Solar Phys. 120, 235

Wu L., Ai G., 1990, Acta. Astron. Sin. 10, 371

Wu S.T., Chang H.M., Hagyard M.J., 1985, in Measurements of Solar Vector Magnetic Fields, Hagyard M.J. (ed.). NASA CP-2374, Marshall Space Flight Center Workshop, p. 49

Wu S.T., Sun M.T., Chang H.M., Hagyard M.J., Gary G.A., 1990, ApJ 362, 698

Zhang H., 1993, Solar Phys. 146, 75

Zhang H., 1994, Solar Phys. 154, 207 